\title{
Editorial
}

\section{Cardiovascular Function in Intensive Care Medicine or Homo Mensura Est}

\author{
Mitja Lainscak, ${ }^{1,2}$ Zsolt Molnar, ${ }^{3}$ Xavier Monnet, ${ }^{4,5}$ and Gorazd Voga ${ }^{6}$ \\ ${ }^{1}$ Department of Cardiology and Department of Research and Education, General Hospital Celje, Oblakova 5, 3000 Celje, Slovenia \\ ${ }^{2}$ Faculty of Medicine, University of Ljubljana, Vrazov trg 2, 1000 Ljubljana, Slovenia \\ ${ }^{3}$ Department of Anesthesiology and Intensive Therapy, Faculty of Medicine, University of Szeged, 6 Semmelweis Street, \\ Szeged 6725, Hungary \\ ${ }^{4}$ AP-HP, Hôpitaux Universitaires Paris-Sud, Hôpital de Bicêtre, Service de Réanimation Médicale, 94270 Le Kremlin-Bicêtre, France \\ ${ }^{5}$ Université Paris-Sud, Faculté de Médecine Paris-Sud, Inserm UMR U-999, 94270 Le Kremlin-Bicêtre, France \\ ${ }^{6}$ Medical ICU, General Hospital Celje, Oblakova 5, 3000 Celje, Slovenia
}

Correspondence should be addressed to Mitja Lainscak; mitja.lainscak@guest.arnes.si

Received 31 December 2015; Accepted 2 March 2016

Copyright (c) 2016 Mitja Lainscak et al. This is an open access article distributed under the Creative Commons Attribution License, which permits unrestricted use, distribution, and reproduction in any medium, provided the original work is properly cited.

Global burden of disease, in terms of both mortality and morbidity, is increasing [1,2]. Ageing of population and better management of acute conditions are significant contributors, yet there is much more to be done. Also, there are numerous situations in clinical practice where we are left with limited evidence and various degrees of uncertainty regarding how to deliver the best medical practice to our patients. This in particular applies for emergency and intensive care where decisions need to be taken within minutes if not seconds; it is therefore not surprising that practicing physician may take suboptimal ways to handle the clinical challenges [3]. Heart failure serves as a good example and in fact this is only cardiovascular condition with increasing prevalence [4]. By adding various comorbidities to the main disease (e.g., anaemia, chronic obstructive pulmonary disease, chronic kidney disease, and cancer, all potentially with body wasting), you end up with an individual prone to deterioration of one or more components of its health status [5-9]. An additional challenge in acute deterioration of chronic disease is iatrogenic due to complex pharmacological therapy; in its essence, very best incentive for a stable patient can get really cumbersome for patient and a clinician once faced with failing organ functions, a common scenario in emergency and intensive medicine. With changes in pharmacokinetics, pharmacodynamics effects are unpredictable, as may be the drugdrug and in particular drug-disease or drug-disease-drug interactions [10-12]. Adding the immediate-acting drugs we usually use in this setting, we are indeed exploring the limited-evidence land and outcomes are less predictable. This, in fact, reminds us once again that we need to consider every patient as an individual with peculiarities and specific response to disease/acute condition and management we employ. Herein, we need to get back to the basics or to the fact that Homo mensura est (Homo mensura est stands for man is the measure said by Protagoras (Greek: $\Pi \rho \omega \tau \alpha \gamma$ ó $\rho \alpha \varsigma$, c. $490-$ c. 420 BC)) or, in other words, that medicine is an art [13]. Yet, this coin has two sides: one is the patient whilst the other one is the practicing physician. And both of them are humans, with all pros and cons. Once in hospital due to acute condition or with critical illness, a comprehensive evaluation of cardiovascular system is crucial for reliable assessment of disease severity and management steps. Here, the other side of the coin, namely, the practicing physician, is taking Centre stage. Again, Homo mensura est is more true than ever. With all difficulties to assess the cardiovascular function and to take decisions in best of patient interest, one needs to rely on parameters one can reliably assess and interpret. This largely depends on one's training, experience, and confidence with particular monitoring tools or biomarkers [14-16]. Unfortunately, clinical practice tells us, despite all efforts by the clinical community $[17,18]$, that we do not meet the standards of good clinical practice [19]. Counterintuitively, 
the development of less invasive methods for assessment of haemodynamic parameters (that all have limitations in the critically ill) did not change patterns of invasive haemodynamic monitoring. Despite availability, echocardiography remains critically underused for these purposes. The lesson learned from the FENICE study [19] should be considered as an important signal to fine-tune our preclinical and clinical training to optimize patient assessment and management.

In acute conditions, we are indeed left with limitedevidence-based medicine. But are there ways to overcome this? Primarily, we should not have prejudices how to handle our patients. The story of beta-blockers in heart failure might serve as a useful example. Initially these drugs were contraindicated, but in current heart failure guidelines, the largest wealth of evidence for prognostic benefit lies within them. Indeed, we are trying to break some long-lasting taboos of misconception through use of these pharmacological agents in obstructive pulmonary disease and during acute deterioration [20-23]. A similar frontier is sepsis, for example, [24]. In sepsis, a closer cooperation between scientific communities, clinicians, and regulatory agencies is required in order to meet future challenges. Some other communities have already paved the way and it is our mission to follow in their footsteps [25]. This issue tried to address some of these aspects. Indeed, we feel that education aiming to optimize patient assessment and to understand the pathophysiological rationale of our actions is crucial in our striving to improve patient outcome. In clinical practice, various options for haemodynamic assessment should be available. Accurate measurement gives us reliable findings that are the basis for timely diagnosis and therapeutic decisions, tailored to individual patient. We would therefore like to promote this special issue and the two review articles by J. Benes et al. and H. A. Gaspar and S. S. Morhy in particular; furthermore, we would once again like to underline the Protagoras quote Homo mensura est. Although generally applicable, the significance in emergency and intensive care medicine may be particularly relevant.

Mitja Lainscak
Zsolt Molnar
Xavier Monnet
Gorazd Voga

\section{References}

[1] T. Vos, R. M. Barber, and B. Bell, "Global, regional, and national incidence, prevalence, and years lived with disability for 301 acute and chronic diseases and injuries in 188 countries, 19902013: a systematic analysis for the Global Burden of Disease Study 2013," The Lancet, vol. 386, no. 9995, pp. 743-800, 2015.

[2] M. Naghavi, H. Wang, and R. Lozano, "Global, regional, and national age-sex specific all-cause and cause-specific mortality for 240 causes of death, 1990-2013: a systematic analysis for the Global Burden of Disease Study 2013," The Lancet, vol. 385, no. 9963, pp. 117-171, 2015.

[3] J.-L. Vincent, J. B. Hall, and A. S. Slutsky, "Ten big mistakes in intensive care medicine," Intensive Care Medicine, vol. 41, no. 3, pp. 505-507, 2015.
[4] P. Ponikowski, S. D. Anker, K. F. AlHabib et al., "Heart failure: preventing disease and death worldwide," ESC Heart Failure, vol. 1, no. 1, pp. 4-25, 2014.

[5] A. P. Maggioni, S. D. Anker, U. Dahlström et al., "Are hospitalized or ambulatory patients with heart failure treated in accordance with European Society of Cardiology guidelines? Evidence from 12440 patients of the ESC Heart Failure LongTerm Registry," European Journal of Heart Failure, vol. 15, no. 10, pp. 1173-1184, 2013.

[6] S. Von Haehling, J. C. Schefold, L. Majc Hodoscek et al., "Anaemia is an independent predictor of death in patients hospitalized for acute heart failure," Clinical Research in Cardiology, vol. 99, no. 2, pp. 107-113, 2010.

[7] R. Marcun, A. Sustic, P. M. Brguljan et al., "Cardiac biomarkers predict outcome after hospitalisation for an acute exacerbation of chronic obstructive pulmonary disease," International Journal of Cardiology, vol. 161, no. 3, pp. 156-159, 2012.

[8] J. C. Schefold, J. Bierbrauer, and S. Weber-Carstens, "Intensive Care Unit-Acquired Weakness (ICUAW) and muscle wasting in critically ill patients with severe sepsis and septic shock," Journal of Cachexia, Sarcopenia and Muscle, vol. 1, no. 2, pp. 147-157, 2010.

[9] S. D. Anker and S. von Haehling, "Efforts begin to sprout: publications in JCSM on cachexia, sarcopenia and muscle wasting receive attention," Journal of Cachexia, Sarcopenia and Muscle, vol. 5, no. 3, pp. 171-176, 2014.

[10] K. Trobec, M. K. Kos, S. Von Haehling, J. Springer, S. D. Anker, and M. Lainscak, "Pharmacokinetics of drugs in cachectic patients: a systematic review," PLoS ONE, vol. 8, no. 11, Article ID e79603, 2013.

[11] T. Roblek, K. Trobec, A. Mrhar, and M. Lainscak, "Potential drug-drug interactions in hospitalized patients with chronic heart failure and chronic obstructive pulmonary disease," Archives of Medical Science, vol. 10, no. 5, pp. 920-932, 2014.

[12] T. Roblek, T. Vaupotic, A. Mrhar, and M. Lainscak, "Drug-drug interaction software in clinical practice: a systematic review," European Journal of Clinical Pharmacology, vol. 71, no. 2, pp. 131-142, 2015.

[13] K. Bendjelid, B. Levy, and A. Broccard, "Intensive care medicine science: an art based on applied physiology?" BioMed Research International, vol. 2015, Article ID 479134, 2 pages, 2015.

[14] Z. Molnár and J.-L. Vincent, "Still a (valuable) place for the pulmonary artery catheter," International Journal of Cardiology, vol. 173, no. 2, pp. 131-132, 2014.

[15] X. Monnet and J.-L. Teboul, "Assessment of volume responsiveness during mechanical ventilation: recent advances," Critical Care, vol. 17, no. 2, article 217, 2013.

[16] X. Monnet, A. Bataille, E. Magalhaes et al., "End-tidal carbon dioxide is better than arterial pressure for predicting volume responsiveness by the passive leg raising test," Intensive Care Medicine, vol. 39, no. 1, pp. 93-100, 2013.

[17] B. P. Cholley, P. H. Mayo, J. Poelaert et al., "Expert round table on ultrasound in icu, international expert statement on training standards for critical care ultrasonography," Intensive Care Medicine, vol. 37, no. 7, pp. 1077-1083, 2011.

[18] J. D. Wilde, J. F. Bion, A. Bullock et al., "The educational environment for training in intensive care medicine: structures, processes, outcomes and challenges in the European region," Intensive Care Medicine, vol. 35, no. 9, pp. 1575-1583, 2009.

[19] M. Cecconi, C. Hofer, J.-L. Teboul et al., "Fluid challenges in intensive care: the FENICE study: a global inception cohort study," Intensive Care Medicine, vol. 41, no. 9, pp. 1529-1537, 2015. 
[20] M. Lainscak, M. Podbregar, D. Kovacic, J. Rozman, and S. von Haehling, "Differences between bisoprolol and carvedilol in patients with chronic heart failure and chronic obstructive pulmonary disease: a randomized trial," Respiratory Medicine, vol. 105, supplement 1, pp. S44-S49, 2011.

[21] M. Lainscak, L. M. Hodoscek, H.-D. Düngen et al., "The burden of chronic obstructive pulmonary disease in patients hospitalized with heart failure," Wiener Klinische Wochenschrift, vol. 121, no. 9-10, pp. 309-313, 2009.

[22] J. T. Parissis, C. Andreoli, N. Kadoglou et al., "Differences in clinical characteristics, management and short-term outcome between acute heart failure patients chronic obstructive pulmonary disease and those without this co-morbidity," Clinical Research in Cardiology, vol. 103, no. 9, pp. 733-741, 2014.

[23] M. Lainscak and S. D. Anker, "Heart failure, chronic obstructive pulmonary disease, and asthma: numbers, facts, and challenges," ESC Heart Failure, vol. 2, no. 3, pp. 103-107, 2015.

[24] A. Morelli, C. Ertmer, M. Westphal et al., "Effect of heart rate control with esmolol on hemodynamic and clinical outcomes in patients with septic shock: a randomized clinical trial," The Journal of the American Medical Association, vol. 310, no. 16, pp. 1683-1691, 2013.

[25] K. Fearon, J. Argiles, V. Baracos et al., "Request for regulatory guidance for cancer cachexia intervention trials," Journal of Cachexia, Sarcopenia and Muscle, vol. 6, no. 4, pp. 272-274, 2015. 


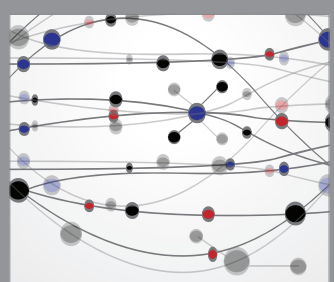

The Scientific World Journal
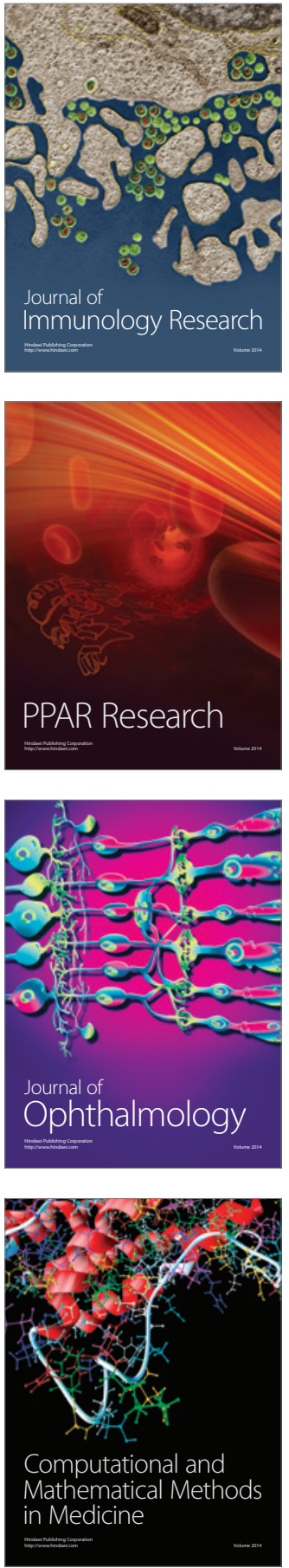

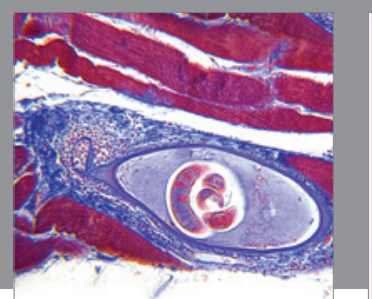

Gastroenterology Research and Practice

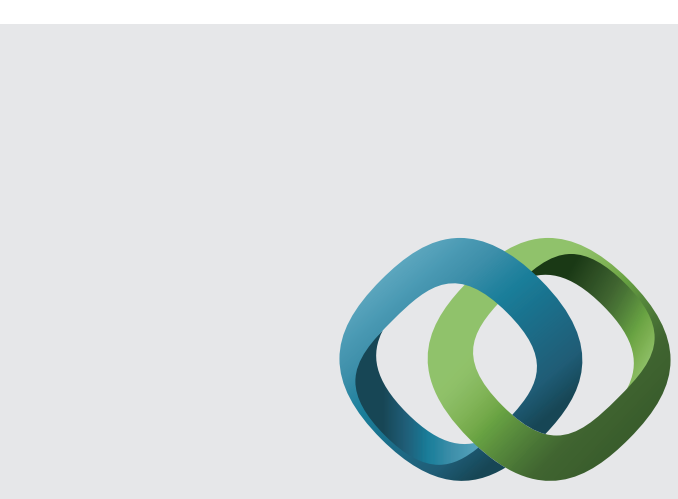

\section{Hindawi}

Submit your manuscripts at

http://www.hindawi.com
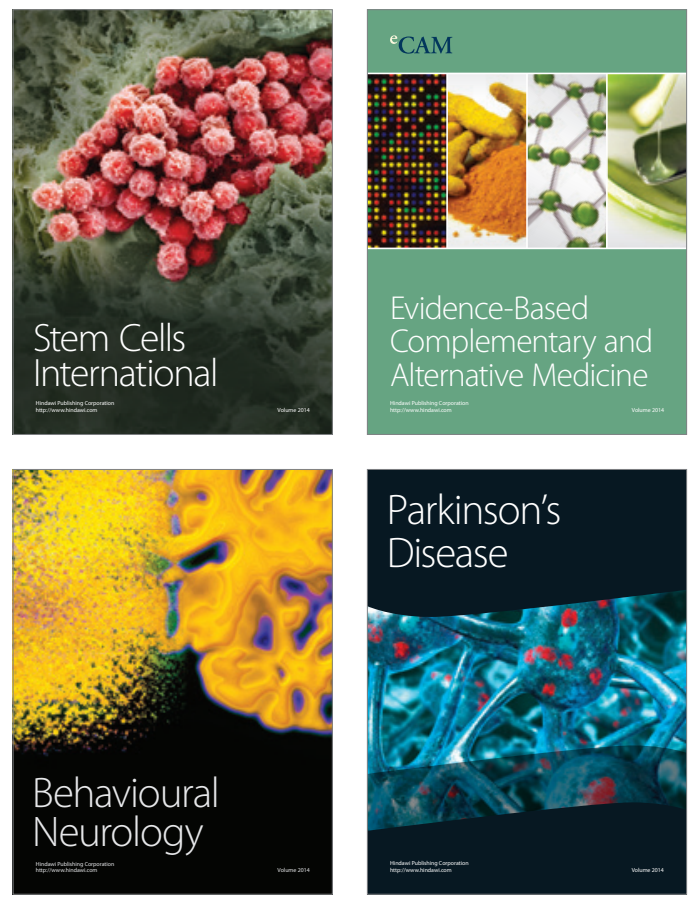
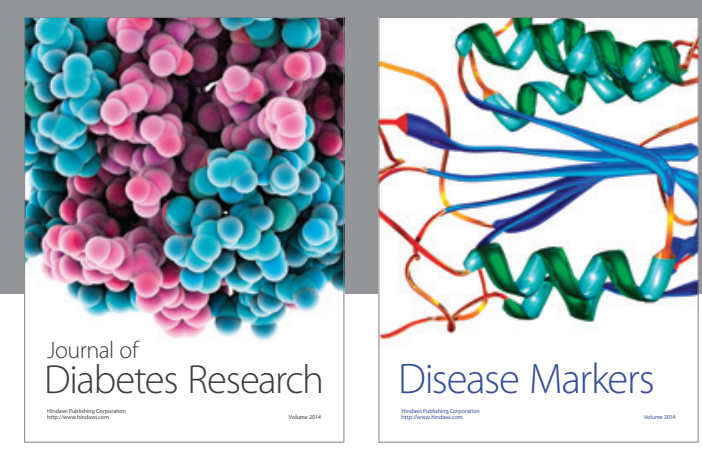

Disease Markers
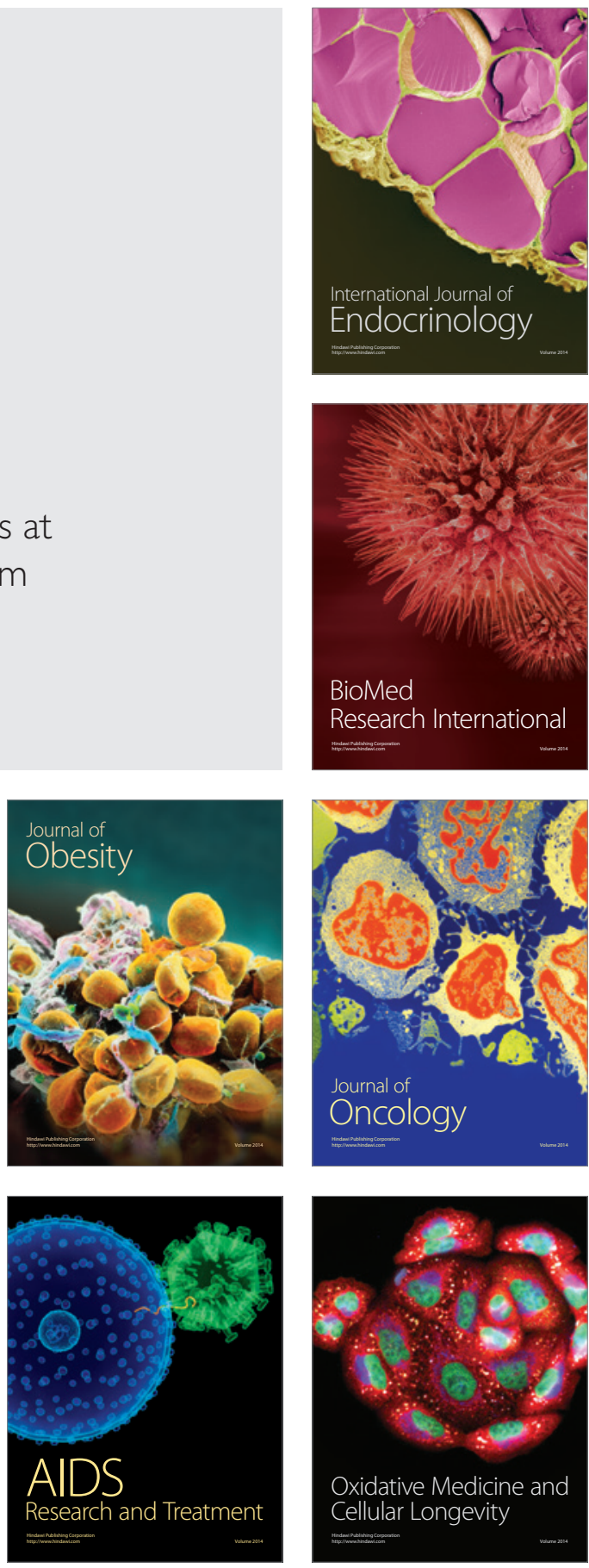